\title{
Effect of Roll Motion Control on Vehicle Lateral Stability and Rollover Avoidance *
}

\author{
Abbas Chokor, Reine Talj, Moustapha Doumiati and Ali Charara
}

\begin{abstract}
This paper discusses the effects of the roll control on the vehicle performance. Rollover avoidance and lateral stability constitute the core analysis of this paper. Two roll reference generators, one static (towards zero) and one dynamic (function of the vehicle lateral acceleration) are designed for control purpose. Roll motion control is achieved through the generation of a feedback roll moment. To track the static roll reference, the roll moment can be allocated to the active suspensions, the semi-active suspensions, or the active antiroll bar, while the roll motion control towards the dynamic reference can be only achieved using the active suspensions. To do so, firstly, based on the time-domain equations of motion of the full-vehicle nonlinear model, a study on how the roll control can help the vehicle to avoid the rollover without deceleration or steering actions is done. Secondly, a frequency analysis of the lateral stability response to the steering input, with and without roll motion control is performed to extract the ranges of steering frequencies and amplitudes where the roll control could be useful. For this study, two lateral-roll linear time invariant vehicle models (without and with linear quadratic roll control) are compared. Thirdly, two robust roll controllers, i.e., Lyapunov-based, and super-twisting sliding mode are developed, validated and compared on the full vehicle nonlinear model using Matlab/Simulink. This paper also provides a comparison between the roll angle control towards the static and the dynamic references.
\end{abstract}

\section{INTRODUCTION}

Driving safety is a major challenge where rollover and lateral skidding commit the major fatal injuries [1], [2]. The effect of the roll motion control on the rollover phenomenon is obvious since it is effected by the lateral acceleration. The vehicle rollover has been treated by several Advanced Driving Assistance Systems (ADAS), either by braking or steering [3], [4], or in a Global Chassis Control $(G C C)$ architectures [5], [6], [7] where the authors propose Direct Yaw Controllers $(D Y C)$ that has the desired yaw rate to switch between two expressions, one is to enhance the lateral stability and the other one is to avoid rollover. However, these systems have the disadvantage of decelerating the vehicle. Authors in [5] have shown a better performance by achieving the same $D Y C$ objective through the Active Differential Braking $(A D B)$. Some other relevant research such as [3], [4] propose to control the roll motion by the Active Front Steering $(A F S)$ and/or $A D B$ to avoid the rollover, regardless of the vehicle maneuverability and trajectory.

\footnotetext{
* This work was supported by the Hauts-de-France Region and the European Regional Development Fund (ERDF).

A. Chokor, R. Talj and A. Charara are with Sorbonne universités, Université de Technologie de Compiègne, CNRS, Heudiasyc UMR 7253, CS 60 319, 60203 Compiègne, France.

M. Doumiati is with ESEO-IREENA EA 4642, 10 Bd Jeanneteau, 49100 Angers, France.
}

The effect of the roll motion control on the lateral stability is more complicated. The dynamics coupling between the vertical and lateral tire forces is an essential key to enhance the lateral stability [8]. Several studies on the (semi-)Active Suspensions (ASus) are conducted to explicitly try to enhance the lateral stability [9], [10]. The basic idea is to prevent the saturation of tires lateral forces. For that, researchers as in [12], [13], [14] propose to control the vertical load transfer when cornering. However, this method may ensure a posterior enhancement on the lateral stability but not a prior demonstrated guaranty. One of this paper objectives is to demonstrate, a priori, in the frequency domain, that the roll angle control can always enhance the lateral stability.

Therefrom, the noticed enhancements in these different $G C C$ approaches have motivated us to create a new synergy represented by achieving the rollover avoidance and the lateral stability objectives through the ASus, usually used for ride comfort and road holding.

The main contributions of this paper are:

- a time-domain analysis of the effect of the roll motion control on the rollover avoidance;

- a frequency-domain analysis of the effect of the roll motion control on the lateral stability;

- development and comparison between a Linear Quadratic Regulator $(L Q R)$, a Lyapunov-based, and a Super-Twisting Sliding Mode (STSM) controllers to control the roll motion;

- a comparison between the roll angle control towards the static reference zero and the new desired roll angle (dynamic reference).

The paper structure is as follows: Section II provides a nonlinear representation of the vehicle roll motion dynamics, followed by a linear vehicle model called extended bicycle model. Section III and IV expose the roll control effect on rollover and lateral stability problems. Section V presents the general closed loop architecture, including the reference generator, the controllers and the actuators. Section VI exposes the Lyapunov-based and the STSM controllers. In Section VII, the proposed controllers are validated by simulation. Finally, Section VIII concludes the achievements of this work and provides a glance about future contributions.

\section{VEHICLE DYNAMICS}

A full vehicle model has been already developed and validated using "SCANeR Studio Simulator" [15], [16]. The full vehicle model serves here to validate the roll motion controllers. From the full vehicle model, the dynamic equation 


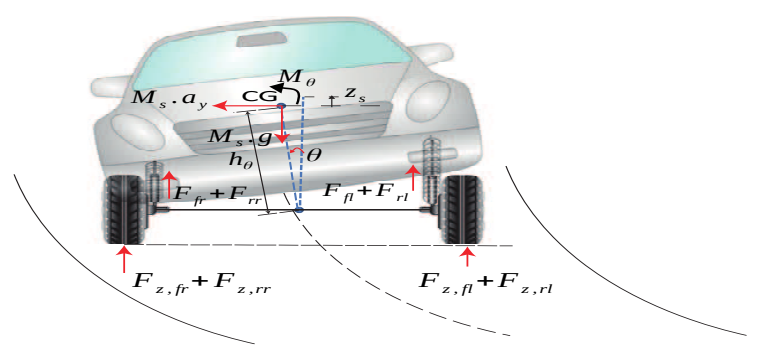

Fig. 1: roll motion (front view)

of the sprung mass roll motion is modeled as:

$$
\begin{aligned}
\ddot{\theta} & =\frac{1}{I_{x}+M_{s} h_{\theta}^{2}}\left[\left(-F_{f r}+F_{f l}\right) t_{f}+\left(-F_{r r}+F_{r l}\right) t_{r}\right. \\
& \left.+M_{s}\left(h_{\theta} \cos (\theta)+z_{s}\right) a_{y}+M_{s}\left(h_{\theta} \sin (\theta)+z_{s}\right) g+M_{\theta}\right],
\end{aligned}
$$

as shown in Fig. 1, where, $M_{s}, h_{\theta}$ and $h_{\phi}$ are respectively the sprung mass weight, the distance between the center of gravity of the sprung mass and the roll rotation center, and the distance between the center of gravity of the sprung mass and the pitch rotation center. $I_{x}$ is the moment of inertia of the sprung mass around the $x$ axis. $a_{y}$ is the vehicle lateral acceleration, considered as exogenous inputs to this equation. $M_{\theta}$ is the active roll moment to be generated through the ASus forces, and $F_{i j}$ is the passive suspension force on the vehicle corner $i j(i=\{f:$ front $, r:$ rear $\}$ and $j=\{r:$ right,$l$ : left $\}$ ), such as:

$$
F_{i j}=-K_{s, i j}\left(z_{s, i j}-z_{u s, i j}\right)-C_{s, i j}\left(\dot{z}_{s, i j}-\dot{z}_{u s, i j}\right),
$$

where $K_{s, i j}$, and $C_{s, i j}$ are respectively the suspension stiffness coefficient and the suspension damping coefficient. $z_{s, i j}$ and $z_{u s, i j}$ are respectively the vertical displacement of the sprung mass and the unsprung mass (wheel bounce), considered as exogenous inputs to this equation.

From the other side, the extended bicycle model is a coupled lateral-roll vehicle model. It is a linear simplified vehicle model which combines the vehicle yaw and side-slip to the roll motion. This model is suitable to analyze the effect of the roll motion control on the lateral stability of the vehicle. The extended bicycle model is inspired from literature [12] as the following:

$$
\begin{aligned}
I_{z} \ddot{\psi} & =F_{y f} l_{f}+F_{y r} l_{r}+I_{x z} \ddot{\theta}, \\
M V(\dot{\beta}+\dot{\psi}) & =F_{y f}+F_{y r}+M_{s} h_{\theta} \ddot{\theta}, \\
\left(I_{x}+M_{s} h_{\theta}^{2}\right) \ddot{\theta} & =M_{s} h_{\theta} V(\dot{\beta}+\dot{\psi})+\left(M_{s} g h_{\theta}-K_{\theta}\right) \theta-C_{\theta} \dot{\theta}+M_{\theta},
\end{aligned}
$$

where $F_{y f}$ and $F_{y r}$ respectively represent the lateral force of the tire on the front axle and on the rear axle; $M_{\theta}$ represents the active roll moment as a control input. $F_{y f}$ and $F_{y r}$ are supposed to be linear to the wheels side-slip angle such that:

$$
\begin{aligned}
& F_{y f}=\mu C_{f} \alpha_{f}, \\
& F_{y r}=\mu C_{r} \alpha_{r},
\end{aligned}
$$

where $C_{f}$ and $C_{r}$ are respectively the double of the front and rear tires cornering stiffness. The wheels side-slip angles are found using the following equations:

$$
\begin{aligned}
& \alpha_{f}=-\beta-\frac{l_{f} \dot{\psi}}{V}+\delta_{f}, \\
& \alpha_{r}=-\beta+\frac{l_{r} \dot{\psi}}{V} .
\end{aligned}
$$

$$
\left[\begin{array}{c}
\dot{\psi} \\
\beta \\
\theta \\
\dot{\theta}
\end{array}\right]=\underbrace{\left(\begin{array}{cccc}
a_{11} & a_{12} & a_{13} & a_{14} \\
a_{21} & a_{22} & a_{23} & a_{24} \\
0 & 0 & 0 & 1 \\
a_{41} & a_{42} & a_{43} & a_{44}
\end{array}\right)}_{A} \underbrace{\left[\begin{array}{c}
\dot{\psi} \\
\beta \\
\theta \\
\dot{\theta}
\end{array}\right]}_{X}+\underbrace{\left(\begin{array}{cc}
b_{u, 11} & b_{u, 12} \\
b_{u, 21} & b_{u, 22} \\
0 & 0 \\
b_{u, 41} & b_{u, 42}
\end{array}\right)}_{B}\left[\begin{array}{c}
\delta_{d} \\
M_{\theta}
\end{array}\right]
$$

As can be seen, the extended bicycle model is an improved version of the bicycle model where firstly the roll dynamics is described by a linear differential equation and secondly it is included into the lateral motion equations. By substituting (5) in (4), and then in (3), the state space representation of the extended bicycle model can be formalized as in (6), where $X=[\dot{\psi}, \beta, \theta, \dot{\theta}]^{T}$ is the state vector. The elements of the state matrix $A \in I R^{4 \times 4}$, and the input matrix $B \in I R^{4 \times 2}$ are formalized in Appendix I.

\section{Roll Motion EfFect on Rollover Problem}

To study the motion roll effect on rollover, suppose that the vehicle has one degree of freedom represented by the roll angle $\theta$ between the suspended and unsuspended masses (see Fig. 1). This means that the suspended mass center of gravity deviates by a positive angle $\theta$ (toward the outside) around the roll axis. Hereby, the moment of all forces around the axis joining outer wheels becomes:

$$
M_{s} a_{y} h-M_{s} g\left(t_{f}-\left(h-h_{r}\right) \sin \theta\right)+F_{z i} 2 t_{f}=0 .
$$

where $F_{z i}$ is the vertical force representing the summation of the front and rear vertical forces of the inner ( w.r.t the corner) wheels, and $t_{f}$ is the vehicle half track. Because of the equilibrium of (7), if $a_{y}$ increases, a natural decreasing of the other single variable $F_{z i}$ happens, up to a certain amount of $a_{y}$ where $F_{z i}$ becomes 0 , which represents inner wheels lift-off. Under the assumption of small angles $\sin \theta \approx \theta$, the lateral acceleration that causes wheels lift-off is:

$$
a_{y, l i f t-o f f}=\frac{t_{f}-\left(h-h_{r}\right) \theta}{h} g,
$$

In order to stay in a safe driving region, a safety factor of 0.7 of the total $a_{y, \text { lift-off }}$ expressed in (8) is proposed, such as:

$$
a_{y, \text { safe }}=0.7 a_{y, l i f t-o f f}=0.7 \frac{t_{f}-\left(h-h_{r}\right) \theta}{h} g
$$

To avoid the rollover, $a_{y}$ should be maintained below $a_{y_{s a f e}}$. While several control actions like the $A F S$ and the $A D B$ aim to reduce $a_{y}$, the $A S u s$, semi-ASus and the Active antiRoll Bar $(A R B)$ aim, by controlling the roll angle, to elevate the maximal safe lateral acceleration $a_{y, \text { safe }}$. For instance, stiffening the suspensions using the semi-ASus or the $A R B$ can reduce the vehicle roll angle towards zero which arises $a_{y, \text { safe }}$ as exhibits equation (9). The contribution that adds the ASus system is the ability to continue turning the roll angle in the negative direction (to the inner side of the corner), that means $a_{y, \text { safe }}$ will be more shifted to a higher value. The choice of the desired roll angle $\theta_{\text {des }}$ is done as follows: - At zero lateral acceleration (straight road), the desired roll angle is $0^{\circ}$.

- At a lateral acceleration equal to the maximal static safe lateral acceleration threshold $0.7 \frac{t_{f}}{h} g$, the desired roll angle 
is equal to the maximal achievable roll angle $10^{\circ}$ (vehicle design constraints) [17].

- The map between $\theta_{d e s}$ and $a_{y}$ is supposed to be linear to make a smooth comfortable roll change rate. Thus, the desired roll angle $\theta_{\text {des }}$ is given as:

$$
\theta_{\text {des }}=-\frac{10 \frac{\pi}{180}}{0.7 \frac{t_{f}}{h} g} a_{y} .
$$

\section{Roll Control EFFect on LATERAL Stability}

\section{A. Stability Index SI Criterion}

The most known criterion to evaluate the lateral stability is called "Stability Index" $(S I)$ [18], [19], [20]. SI can be expressed as:

$$
S I=\left|q_{1} \beta+q_{2} \dot{\beta}\right|,
$$

where $q_{1}$ and $q_{2}$ are identified depending on the vehicle parameters and road adherence $\mu$ to characterize the stable boundary of the $\beta-\dot{\beta}$ phase plane. $S I$ is normalized and varies between 0 and 1 . For $S I \leq \underline{S I}$ (a predefined lower threshold depending on the vehicle and road parameters), the vehicle is in normal driving situations (stable region); up to a predefined higher threshold $\overline{S I}$, the vehicle is considered in the critical lateral stability region, where active safety controllers have to be triggered to cover back the lateral stability of the vehicle; beyond $\overline{S I}$ the vehicle operates in the unstable region.

\section{B. Frequency Analysis Setup}

The relation between the roll angle $\theta$ and the lateral stability quantified by the $S I$ criterion $(\beta-\dot{\beta})$ is governed by the dynamical system given in (6). Therefore, this section analyzes, in the frequency and time domains, the effect of the roll control on the lateral stability.

The LTI model (6) can be written as:

$$
\dot{X}=A X+B_{1} \delta_{d}+B_{2} M_{\theta},
$$

where $B_{1}$ and $B_{2}$ are respectively the first and second column of $B$.

The objective is to compare the frequency response of the vehicle with a controlled roll motion as in (12), and the vehicle without a roll controller $\left(M_{\theta}=0\right)$ as in (13):

$$
\dot{X}=A X+B_{1} \delta_{d} .
$$

Thus, let consider the state feedback $L Q R$ control law:

$$
M_{\theta}=-K X .
$$

The optimal closed-loop system becomes:

$$
\dot{X}=\left(A-B_{2} K\right) X+B_{1} \delta_{d},
$$

which has the same form as (13), but with a controlled roll motion.

The optimization procedure consists in finding the control input $U=M_{\theta}$ which minimizes the performance index $J$ :

$$
J=\int_{0}^{\infty}\left(X^{T} Q X+U^{T} R U\right) d t
$$

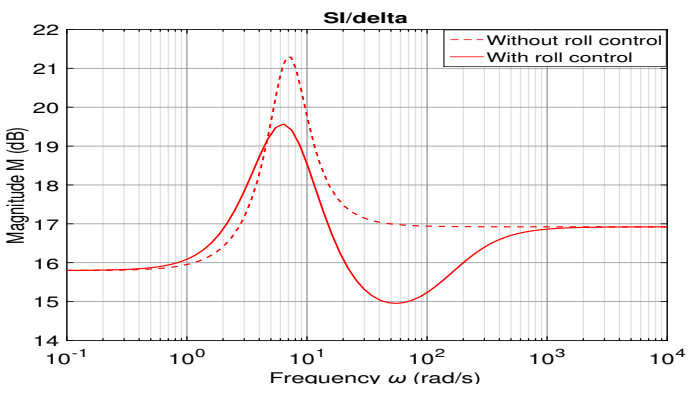

Fig. 2: Bode plot $S I / \delta_{d} ; V=100 \mathrm{~km} / \mathrm{h}$

where $Q$ and $R$ are the weighting matrices.

The control purpose is to minimize the roll angle and roll velocity, by controlling $M_{\theta}$. Thus, the performance index $J$ becomes:

$$
J=\int_{0}^{\infty}\left(\rho_{1} \theta^{2}+\rho_{2} \dot{\theta}^{2}+M_{\theta}^{2}\right) d t,
$$

where the weighting coefficients $\rho_{1}$ and $\rho_{2}$ are adjusted to promote the weight on the roll angle and its rate of change, while minimizing the energy of the control input.

The matrix gain $K$ has the form:

$$
K=R^{-1} B_{2}^{T} P,
$$

where the matrix $P$ is the solution of the Algebric Riccati Equation:

$$
A^{T} P+P A-P B_{2} R^{-1} B_{2}^{T} P+Q=0 .
$$

The frequency response of the vehicle with roll controller (15) and the vehicle without roll controller (13) can be now compared.

\section{Lateral Stability Frequency Analysis}

To analyze the frequency response of the vehicle lateral stability w.r.t the driver steering input, the transfer functions $G_{\delta_{d}}^{S I}$ of both uncontrolled (13) and controlled (15) LTI systems are evaluated in Matlab environment, and their bode diagrams at the vehicle speed $V=100 \mathrm{~km} / \mathrm{h}$ are plotted in Fig. 2. Both curves in Fig. 2 represent the magnitude $M(\omega)$ of $G_{\delta_{d}}^{S I}$ in $(d b)$ over the frequency range $\omega$ of $\delta_{d}$.

The magnitude response of $G_{\delta_{d}}^{S I}$ of the uncontrolled roll vehicle increases significantly, while controlling the roll angle remarkably reduces the response of $G_{\delta}^{S I}$, especially at frequencies around the peak magnitude. Based on the following relation,

$$
S I=A * 10^{\frac{M(\omega)}{20}},
$$

at a high steering amplitude $(A>0.1 \mathrm{rad})$, the $S I$ of the uncontrolled roll vehicle exceeds $S I=1$, while the controlled one establishes acceptable behavior.

In order to generalize for any speed, the frequency responses $G_{\delta_{d}}^{S I}$ of the controlled and uncontrolled vehicles are evaluated at different speeds $V=70,85,100,115 \mathrm{~km} / \mathrm{h}$. The results are illustrated in Fig. 3. This figure shows that as much as the speed becomes higher, as the difference between the magnitude curves (at the same speed) becomes greater. 


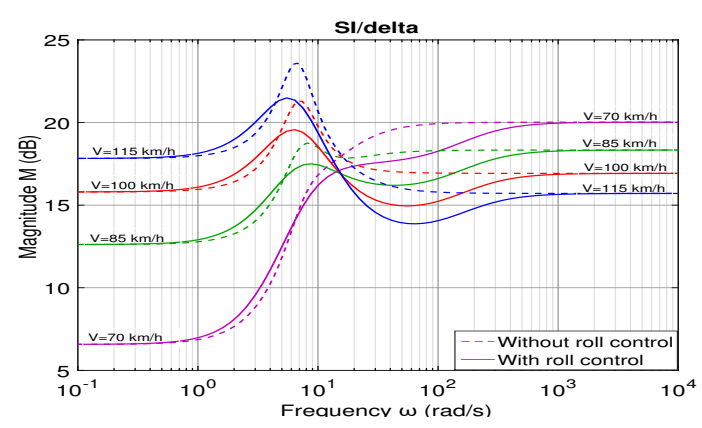

Fig. 3: Bode plot $S I / \delta_{d}$

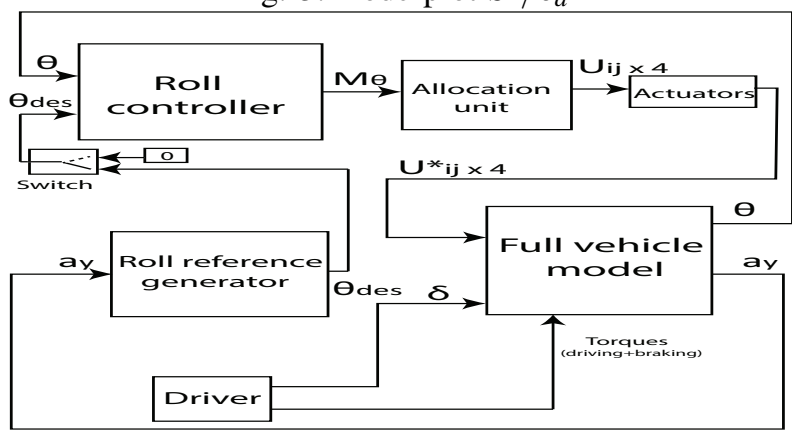

Fig. 4: Control scheme

Hence, at high speed, the roll motion control becomes more influencing on the lateral stability enhancement.

\section{Closed-Loop Control Architecture}

Based on the previous analysis, this section presents the general closed-loop control scheme to be developed as shown in Fig. 4. The roll reference generator block is either static at zero or dynamic as in (10). The roll controller block is one of the three control techniques: Linear Quadratic Regulation $L Q R$, Lyapunov-based, and STSM that will be developed in the next section. The control input $M_{\theta}$ has to be generated by the actuators forces or torques depending on the integrated technology. An example (but not restricted to) is the distribution of $M_{\theta}$ between the four active forces $U_{i j}$ of the ASus. This is done in the control allocation unit as described in (21):

$$
\begin{aligned}
& U_{f l}=0.5 \frac{l_{r}}{l_{f}+l_{r}} \frac{M_{\theta}}{t_{f}} ; U_{f r}=-0.5 \frac{l_{r}}{l_{f}+l_{r}} \frac{M_{\theta}}{t_{f}} ; \\
& U_{r l}=0.5 \frac{l_{f}}{l_{f}+l_{r}} \frac{M_{\theta}}{t_{r}} ; U_{r r}=-0.5 \frac{l_{f}}{l_{f}+l_{r}} \frac{M_{\theta}}{t_{r}} .
\end{aligned}
$$

The choice of this distribution is done in order to avoid any influence on the pitch angle and the bounce displacement as shown in Fig. 5.

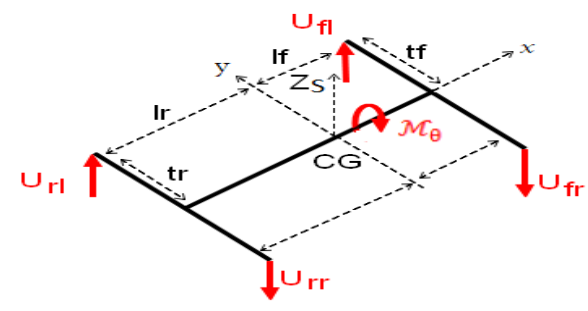

Fig. 5: Active forces distribution

\section{Roll Motion Controllers Design}

This section is dedicated to design robust controllers for the roll motion. The Lyapunov-based control technique and the STSM control technique are used to develop the roll motion controllers. These robust control techniques are chosen to deal with the nonlinear behavior/dynamics of the vehicle. A performance comparison between the Lyapunovbased, the $S T S M$, and the $L Q R$ controllers is also performed. As discussed before, the objectives of these controllers are either to converge the nonlinear roll motion $\theta$ given in (1) to zero or to $\theta_{\text {des }}$ given in (10). The controllers will be developed for a general reference $\theta_{\text {des }}$ of $\theta$, then, they will be tested for both references.

Let first define:

$$
e_{\theta}=\theta-\theta_{\text {des }}
$$

the error between the actual and desired roll angles.

\section{A. Lyapunov-Based Controller}

The control objective is to converge the roll error variable $e_{\theta}$ (of relative degree 2 w.r.t the control input $M_{\theta}$ ) to zero. Let define the "off-the-manifold" variable $z_{\theta}$, such as:

$$
z_{\theta}=\dot{e}_{\theta}+k_{1 \theta} e_{\theta}+k_{2 \theta} \int_{0}^{t} e_{\theta} d \tau
$$

Based on Immersion and Invariance approach [21], the "offthe-manifold" variable $z_{\theta}$ has to converge to the target dynamics corresponding to $z_{\theta}=0$ in the manifold, where the roll error dynamics obey to the following equation:

$$
\dot{e}_{\theta}+k_{1 \theta} e_{\theta}+k_{2 \theta} \int_{0}^{t} e_{\theta} d \tau=0
$$

where $k_{1 \theta}>0$ and $k_{2 \theta}>0$ (Routh-Hurwitz stability condition for a second order characteristic polynomial). Despite the fact that the convergence of $\int_{0}^{t} e_{\theta} d \tau$ to zero is not a necessary condition, its addition helps to reduce the permanent steadystate error.

In order to render the manifold attractive, let define a positive definite Lyapunov candidate function as follows:

$$
V_{\theta}=\frac{1}{2} z_{\theta}^{2}
$$

$\dot{V}_{\theta}$ should be negative (Lyapunov stability conditions), thus,

$$
\dot{V}_{\theta}=z_{\theta} \dot{z}_{\theta} \leq 0,
$$

then, in order to ensure an exponential convergence of $z_{\theta}$ to zero, let:

$$
\dot{z}_{\theta}=-\alpha_{\theta} z_{\theta},
$$

which makes (26) always negative if $\alpha_{\theta}>0$. Thus:

$$
\ddot{e}_{\theta}+k_{1 \theta} \dot{e}_{\theta}+k_{2 \theta} e_{\theta}=-\alpha_{\theta}\left(\dot{e}_{\theta}+k_{1 \theta} e_{\theta}+k_{2 \theta} \int_{0}^{t} e_{\theta} d \tau\right),
$$

where

$$
\ddot{e}_{\theta}=\ddot{\theta}-\ddot{\theta}_{d e s},
$$


then substituting $\ddot{\theta}$ from (1) in (28), the control input $M_{\theta}$ can be found as in (30):

$$
\begin{aligned}
M_{\theta}= & \left(I_{x}+M_{s} h_{\theta}^{2}\right)\left[-M_{\theta_{e q}}+\ddot{\theta}_{\text {des }}-\left(\alpha_{\theta}+k_{1 \theta}\right)\left(\dot{\theta}-\dot{\theta}_{\text {des }}\right)\right. \\
& \left.-\left(\alpha_{\theta} k_{1 \theta}+k_{2 \theta}\right)\left(\theta-\theta_{\text {des }}\right)-\alpha_{\theta} k_{2 \theta} \int_{0}^{t}\left(\theta-\theta_{\text {des }}\right) d \tau\right],
\end{aligned}
$$

where

$$
\begin{aligned}
M_{\theta_{e q}}= & \frac{1}{I_{x}+M_{s} h_{\theta}^{2}}\left[\left(-F_{f r}+F_{f l}\right) t_{f}+\left(-F_{r r}+F_{r l}\right) t_{r}\right. \\
& \left.+M_{s}\left(h_{\theta} \cos (\theta)+z_{s}\right) a_{y}+M_{s}\left(h_{\theta} \sin (\theta)+z_{s}\right) g\right] .
\end{aligned}
$$

This control input means that the vehicle parameters should be well estimated and several variables need to be measured or estimated. Indeed, $-M_{\theta_{e q}}$ and $\left(I_{x}+M_{s} h_{\theta}^{2}\right)$ compensate all the dynamics of the roll angle expressed in (1) as a feedforward command, beside the robust terms of the feedback on $e_{\theta}$, its time derivative and integral of equation (30). $\theta, \dot{\theta}$, and $a_{y}, z_{s}$ are measured by the Inertial Measurement Unit IMU, the suspension forces $F_{i j}$ could be estimated from suspensions' deflections.

\section{B. Super-Twisting Second Order Sliding Mode Controller}

A second controller has been developed based on the STSM technique. The Super-Twisting algorithm is a second order sliding mode control that handles a relative degree equal to one [22]. It generates the continuous control function that drives the sliding variable and its derivative to zero in finite time in the presence of smooth matched disturbances. Let define the sliding variable as follows:

$$
s_{\theta}=\dot{e}_{\theta}+k_{\theta} e_{\theta},
$$

where $k_{\theta}>0$. Unlike the preceding Lyapunov controller, the integral term of $e_{\theta}$ has not been considered inside the variable $s_{\theta}$, because the super-twisting algorithm is a second order sliding mode, it contains an integral term on the sign of $s_{\theta}$ [23]. The variable $s_{\theta}$ has a relative degree of 1 w.r.t the control input $M_{\theta}$, with a second derivative written as:

$$
\ddot{s}_{\theta}\left(s_{\theta}, t\right)=\Phi\left(s_{\theta}, t\right)+\xi\left(s_{\theta}, t\right) \dot{M}_{\theta}(t),
$$

where $\Phi(s, t)$ and $\xi(s, t)$ are unknown bounded functions. The control objective is to achieve the convergence to the sliding surface defined as $s_{\theta}=0$. Only the knowledge of $s_{\theta}$ is required in real time.

Suppose that there exist positive constants $S_{0}, b_{\min }, b_{\max }$, $C_{0}, M_{\theta, \max }$ such that for $\left|s_{\theta}(t)\right|<S_{0}$, the system satisfies the following conditions:

$$
\left\{\begin{array}{l}
\left|M_{\theta}(t)\right| \leq M_{\theta, \max }, \\
\left|\Phi\left(s_{\theta}, t\right)\right|<C_{0}, \\
0<b_{\text {min }} \leq\left|\xi\left(s_{\theta}, t\right)\right| \leq b_{\text {max }} .
\end{array}\right.
$$

The sliding mode control law, based on the Super-Twisting algorithm, is given by:

$M_{\theta}(t)=M_{\theta, 1}+M_{\theta, 2}\left\{\begin{array}{l}\left.\left.M_{\theta, 1}=-\alpha_{\theta}\left|s_{\theta}\right|^{\nabla} \operatorname{sign}\left(s_{\theta}\right) ; \nabla \in\right] 0,0.5\right] \\ \dot{M}_{\theta, 2}=-\beta_{\theta} \operatorname{sign}\left(s_{\theta}\right) .\end{array}\right.$

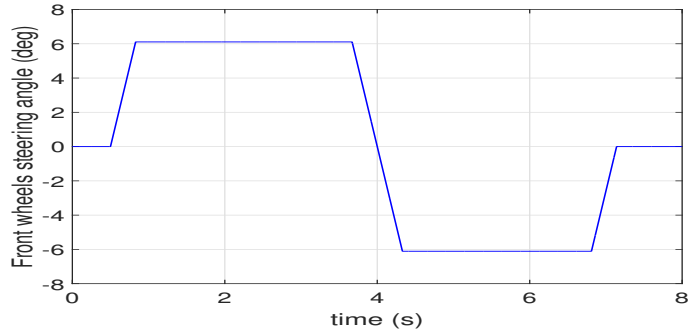

Fig. 6: Fishhook steering

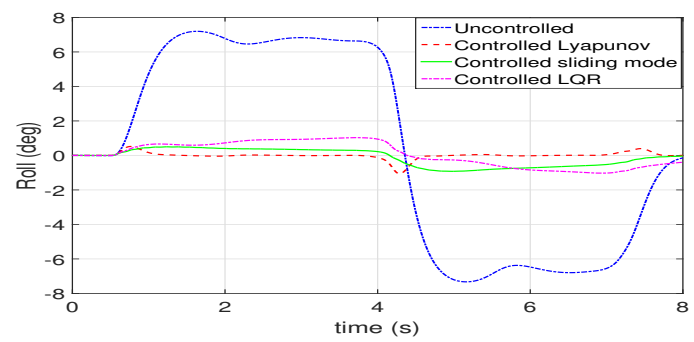

Fig. 7: Roll comparison ; $\theta_{\text {des }}=0$

$\alpha_{\theta}$ and $\beta_{\theta}$ are positive gains. The finite time convergence is guaranteed by the following conditions [23]:

$$
\left\{\begin{array}{l}
\alpha_{\theta} \geq \sqrt{\frac{4 C_{0}\left(b_{\max } \beta_{\theta}+C_{0}\right)}{b_{\min }^{2}\left(b_{\min } \beta_{\theta}-C_{0}\right)}}, \\
\beta_{\theta}>\frac{C_{0}}{b_{\min }} .
\end{array}\right.
$$

The STSM controller is known for its robustness against parameters uncertainties and disturbances. It converges to the sliding surface in finite time. Once $s_{\theta}=0$, the error dynamics obey to the following equation:

$$
\dot{e}_{\theta}+k_{\theta} e_{\theta}=0 .
$$

$\dot{e}_{\theta}$ and $e_{\theta}$ exponentially converge to zero if $k_{\theta}>0$, and the states $\theta$ and $\dot{\theta}$ exponentially converge to $\theta_{d e s}$ and $\dot{\theta}_{d e s}$. To be noted that the term $M_{\theta_{e q}}$ of (31) can be added to the control input $M_{\theta}$, as a feedforward, to achieve a faster convergence towards the sliding surface.

\section{ViI. Controllers Validation and Performance COMPARISON}

In this section, the proposed controllers will be validated on the simulation model (full vehicle model). To do so, the fishhook maneuver shown in Fig. 6 at an initial speed of $V=130 \mathrm{~km} / \mathrm{h}$ is the appropriate test to evaluate both the vehicle rollover risk and the lateral stability.

\section{A. Controllers Validation: static $\theta_{\text {des }}$}

Lyapunov-based, STSM and $L Q R$ controllers are compared in this section to evaluate their performances when minimizing the roll angle to $\theta_{\text {des }}=0$.

Figure 7 shows the roll angle for a vehicle with passive suspensions (uncontrolled roll) which turns up to $7^{\circ}$ in both directions. All the controllers are efficient to control the roll angle toward zero. As a comparison, beside the robust terms of the feedback (30), the Lyapunov-based controller compensates all the dynamics of the roll angle as expressed in (31) to have this performance. That means, in real application, 


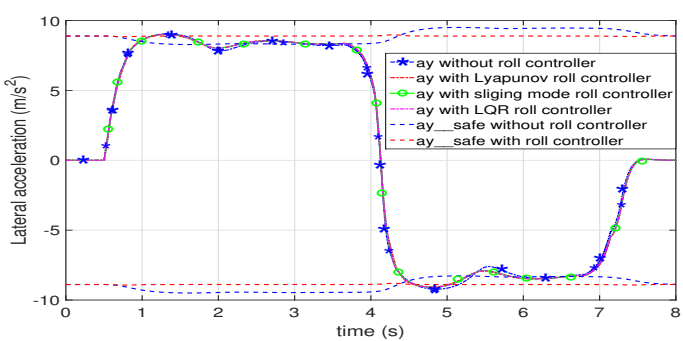

Fig. 8: ay comparison ; $\theta_{\text {des }}=0$

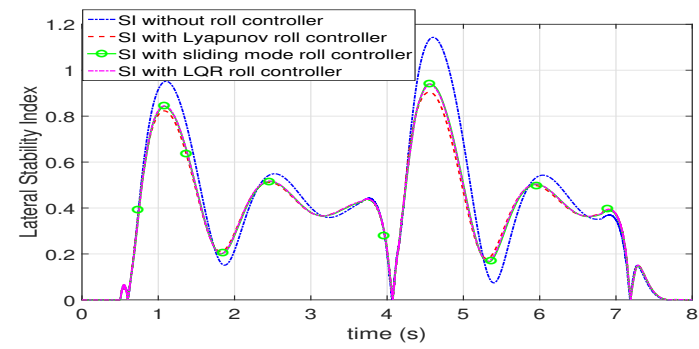

Fig. 9: $S I$ comparison ; $\theta_{\text {des }}=0$

a strong knowledge (estimation, measurement, parameters exactitude, ideal modeling) on the components of $M_{\theta_{e q}}$ is needed. On the other side, the STSM controller is efficient in controlling the roll angle by only the generation of the feedback from the roll angle and velocity (35), with no need to compensate the roll dynamics. The performance of the $L Q R$ controller is also acceptable to control the roll angle towards zero.

Fig. 8 shows the lateral acceleration of the uncontrolled roll vehicle which is approximately the same for all roll controllers. This means that the roll control does not affect the lateral acceleration to avoid rollover. In fact, the controller aims to elevate the maximal safe lateral acceleration expressed in (9), which depends on the roll angle. As the roll angle is minimized to zero, thus, the maximal safe lateral acceleration increases as shown in the same figure. Figure 9 shows the lateral $S I$ of the uncontrolled roll vehicle which exceeds 0.7 , while the controlled ones (by all the proposed controllers) enhance the lateral stability. Even if the enhancement is not sufficient, because the SI remains above 0.7 , the roll control to zero can be used to help the other controllers $(A F S$ and $D Y C)$ to avoid the lateral skidding in a $G C C$ strategy.

\section{B. Controllers Validation: dynamic $\theta_{\text {des }}$}

In this section, the controllers performances will be evaluated when controlling $\theta$ to $\theta_{\text {des }}$ in the opposite direction expressed in (10).

Figure 10 shows the uncontrolled roll angle (same as in Fig. 7), the desired one, and the controlled ones. The Lyapunov-based controlled one, and the STSM controlled one accurately track the desired roll trajectory. The $L Q R$ controller, as is synthesized to minimize the roll angle to zero, not to minimize the error between the roll angle and its desired trajectory, could not track the desired roll as is shown in the same figure. For this reason, in this subsection, the $L Q R$ controller is not compared with the Lyapunov-based

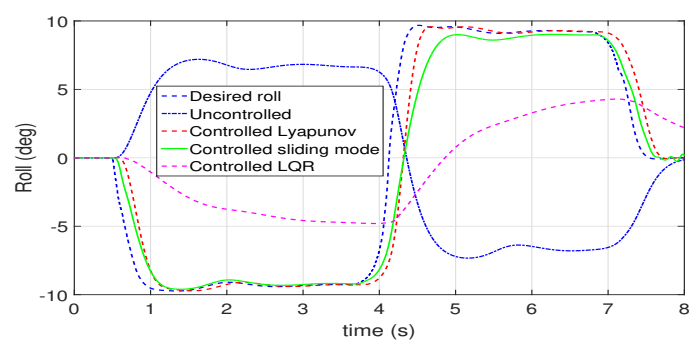

Fig. 10: Roll comparison ; $\theta_{\text {des }}$ opposite direction

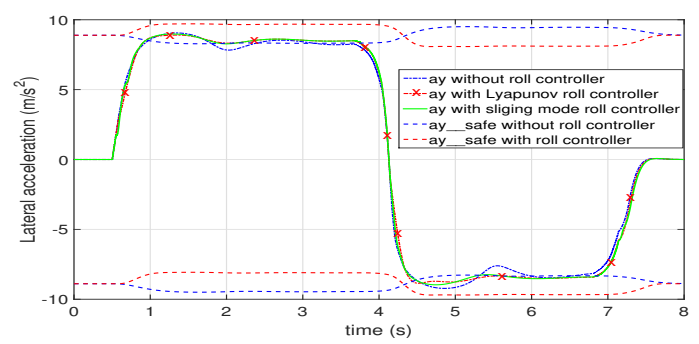

Fig. 11: ay comparison ; $\theta_{\text {des }}$ opposite direction

and STSM controllers.

Figure 11 shows the approximately confounded lateral accelerations of the uncontrolled roll vehicle, and the controlled ones. It also shows the maximal safe lateral acceleration which increases more comparing to the case where the roll angle is minimized to zero. This issue drives away the rollover risk at this range of lateral acceleration. Figure 12 shows the lateral SI of the uncontrolled roll vehicle that exceeds the value 0.7 up to 1.2 which leads the vehicle to loose its lateral stability. Controlling the roll angle toward the inside wheels enhances the lateral stability as shown in the same figure, especially after a sharp steering where the SI is reduced to less than 0.7 by both controllers.

Figure 13 shows the control inputs for both Lyapunovbased and sliding mode controllers which are in fact the ASus forces provided by the actuators (after saturating and filtering). This figure shows that their maximal values are around $4000 \mathrm{~N}$ which is feasible by the ASus actuators without any saturation. This fact makes these developed forces realistic and applicable to the vehicle after controlling the ASus actuators.

\section{Roll Reference Performance Comparison}

Turning the roll angle in the opposite direction requires more energy than just minimizing it to zero, because it can be only achieved by the ASus which consume more energy comparing to the semi-ASus or the $A R B$. This fact has made

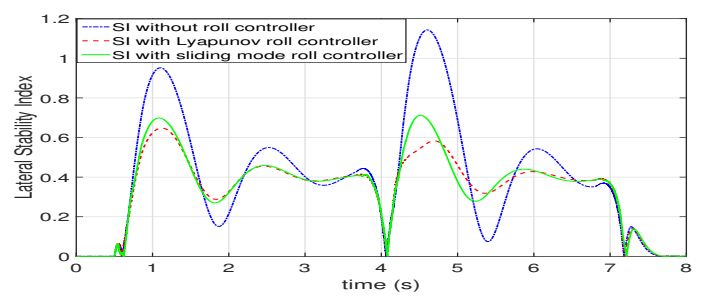

Fig. 12: $S I$ comparison ; $\theta_{\text {des }}$ opposite direction 


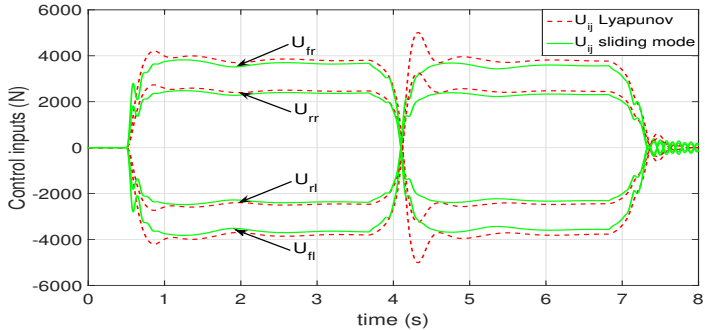

Fig. 13: Control inputs comparison ; $\theta_{\text {des }}$ opposite direction

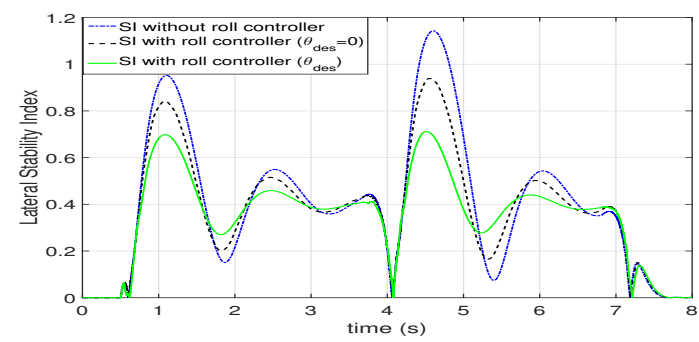

Fig. 14: $S I$ comparison; $\theta_{\text {des }}=0$ vs $\theta_{\text {des }}$ opposite direction

from the ASus a non preferable system to be integrated into series vehicles. However, the results of this paper show better performance in maintaining the lateral stability and avoiding the rollover when turning the roll angle in the opposite direction through the ASus. Figure 14 compares the SI of the vehicle when controlling the roll angle toward zero and toward $\theta_{\text {des }}$ of eq. (10) based on STSM control technique. This figure also shows that the control of the roll angle in the opposite direction brings more enhancement on the lateral stability than just minimizing it to zero. Hence, it could contribute to reduce the energy consumption of other actuators used for lateral stability ( $A F S, A D B \ldots)$.

\section{CONCLUSION}

This work has shown the enhancement that the vehicle roll control could bring on lateral stability and rollover avoidance. Lyapunov-based, STSM, and $L Q R$ roll motion controllers have been developed. Controlling the roll in the opposite direction has demonstrated more advantages on the lateral stability and rollover avoidance compared to only minimizing the roll angle to zero. Future works will integrate this strategy inside a global $G C C$ architecture to show its advantages.

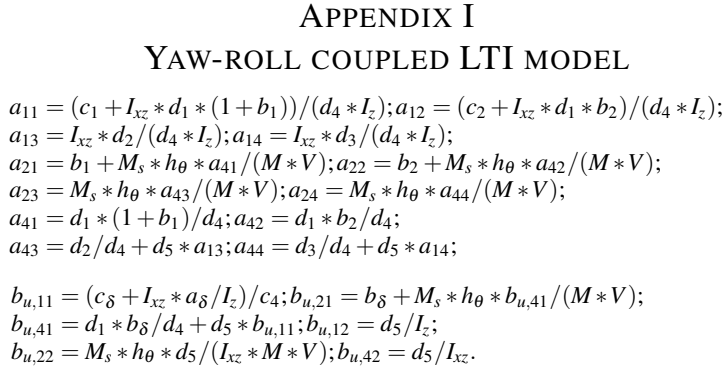

\section{ACKNOWLEDGMENT}

The authors would like to thank the Hauts-de-France Region and the European Regional Development Fund (ERDF) 2014/2020 for the funding of this work, through the SYSCOVI project.

\section{REFERENCES}

[1] H. DOT, "Traffic safety facts research note: summary of statistical findings. highlights of 2009 motor vehicle crashes, dot hs 811363 , washington, dc," 2010

[2] W. Deutermann, "Characteristics of fatal rollover crashes. national center for statistics and analysis, national highway traffic safety administration, nhtsa," Tech. Rep., 2002.

[3] J. Ackermann and D. Odenthal, "Robust steering control for active rollover avoidance of vehicles with elevated center of gravity," Citeseer, 1998.

[4] S. Solmaz, M. Corless, and R. Shorten, "A methodology for the design of robust rollover prevention controllers for automotive vehicles with active steering," International Journal of Control, vol. 80, no. 11, pp. 1763-1779, 2007.

[5] Y. Akhmetov, D. Rémond, L. Maiffredy, M. Di Loreto, W. MarquisFavre, and V. Harth, "Global Chassis Control for active safety of heavy vehicles," in World Automotive Congress FISITA, F2010-D-057, 2010.

[6] M. B. Alberding, J. Tjønnås, and T. A. Johansen, "Nonlinear hierarchical control allocation for vehicle yaw stabilization and rollover prevention," in IEEE European Control Conference (ECC), pp. 4229 4234, 2009.

[7] J. Yoon, S. Yim, W. Cho, B. Koo, and K. Yi, "Design of an unified chassis controller for rollover prevention, manoeuvrability and lateral stability," Vehicle system dynamics, vol. 48, no. 11, pp. 1247-1268, 2010

[8] Y. Li, W. Sun, J. Huang, L. Zheng, and Y. Wang, "Effect of vertical and lateral coupling between tyre and road on vehicle rollover," Vehicle System Dynamics, vol. 51, no. 8, pp. 1216-1241, 2013.

[9] X. Jin, G. Yin, N. Zhang, and J. Chen, "Improving vehicle handling stability performance via integrated control of active front steering and suspension systems," in Transportation Electrification Asia-Pacific (ITEC Asia-Pacific), IEEE Conference and Expo, pp. 621-625, 2016.

[10] S.-e. Zhao, Y. Li, and X. Qu, "Vehicle chassis integrated control based on multimodel and multilevel hierarchical control," Mathematical Problems in Engineering, 2014.

[11] V. T. Vu, O. Sename, L. Dugard, and P. Gáspár, "Enhancing roll stability of heavy vehicle by lqr active anti-roll bar control using electronic servo-valve hydraulic actuators," Vehicle System Dynamics, vol. 55, no. 9, pp. 1405-1429, 2017.

[12] J. Yao, G. Lv, M. Qv, Z. Li, S. Ren, and S. Taheri, "Lateral stability control based on the roll moment distribution using a semiactive suspension," Proceedings of the Institution of Mechanical Engineers, Part D: Journal of Automobile Engineering, vol. 231, no. 12, pp. 1627-1639, 2017.

[13] S. Fergani, L. Menhour, O. Sename, L. Dugard, and B. D’AndréaNovel, "LPV/H $\infty$ suspension robust control adaption of the dynamical lateral load transfers based on a differential algebraic estimation approach," in 8th IFAC International Symposium on Advances in Automotive Control, 2016.

[14] A. Chokor, R. Talj, A. Charara, H. Shraim, and C. Francis, "Active suspension control to improve passengers comfort and vehicle's stability," in 19th IEEE International Conference on Intelligent Transportation Systems (ITSC), pp. 296-301, 2016.

[15] A. Chokor, R. Talj, A. Charara, M. Doumiati, and A. Rabhi, "Rollover prevention using active suspension system," in 20th IEEE International Conference on Intelligent Transportation Systems (ITSC), pp. 17061711, 2017.

[16] A. Y. Ungoren and H. Peng, "Evaluation of vehicle dynamic control for rollover prevention," International Journal of Automotive Technology, vol. 5, no. 2, pp. 115-122, 2004.

[17] S. Inagaki, I. Kshiro, and M. Yamamoto, "Analysis on vehicle stability in critical cornering using phase-plane method," in International Symposium on Advanced Vehicle Control, 1994.

[18] R. Rajamani, Vehicle Dynamics and Control. Springer, 2012.

[19] W. Chen, H. Xiao, Q. Wang, L. Zhao, and M. Zhu, Integrated vehicle dynamics and control. John Wiley \& Sons, 2016.

[20] A. Astolfi, D. Karagiannis, and R. Ortega, Nonlinear and adaptive control with applications. Springer Science \& Business Media, 2007.

[21] Y. Shtessel, C. Edwards, L. Fridman, and A. Levant, Sliding mode control and observation. Springer, 2014.

[22] V. Utkin, "On convergence time and disturbance rejection of supertwisting control," IEEE Transactions on Automatic Control, vol. 58, no. 8, pp. 2013-2017, 2013. 